\title{
Potential Leaders Trade Off Goal-Oriented and Socially Oriented Behavior in Mobile Animal Groups
}

\author{
Christos C. Ioannou, ${ }^{1,2, *}$ Manvir Singh, ${ }^{3}$ and Iain D. Couzin ${ }^{2,4}$ \\ 1. School of Biological Sciences, University of Bristol, Bristol BS8 1TQ, United Kingdom; 2. Department of Ecology and Evolutionary \\ Biology, Princeton University, Princeton, New Jersey 08544; 3. Department of Human Evolutionary Biology, Harvard University, \\ Cambridge, Massachusetts 02138; 4. Department of Collective Behavior, Max Planck Institute for Ornithology, Konstanz, Germany; \\ and Department of Biology, University of Konstanz, D-78457 Konstanz, Germany
}

Submitted November 3, 2014; Accepted February 27, 2015; Electronically published June 5, 2015

Online enhancement: appendix. Dryad data: http://dx.doi.org/10.5061/dryad.qj07b.

ABSTRACT: Leadership is widespread across the animal kingdom. In self-organizing groups, such as fish schools, theoretical models predict that effective leaders need to balance goal-oriented motion, such as toward a known resource, with their tendency to be social. Increasing goal orientation is predicted to increase decision speed and accuracy, but it is also predicted to increase the risk of the group splitting. To test these key predictions, we trained fish (golden shiners, Notemigonus crysoleucas) to associate a spatial target with a food reward ("informed" individuals) before testing each singly with a group of eight untrained fish who were uninformed ("naive") about the target. Informed fish that exhibited faster and straighter paths (indicative of greater goal orientation) were more likely to reach their preferred target and did so more quickly. However, such behavior was associated with a tendency to leave untrained fish behind and, therefore, with failure to transmit their preference to others. Either all or none of the untrained fish stayed with the trained fish in the majority of trials. Using a simple model of self-organized coordination and leadership in groups, we recreate these features of leadership observed experimentally, including the apparent consensus behavior among naive individuals. Effective leadership thus requires informed individuals to appropriately balance goal-oriented and socially oriented behavior.

Keywords: leadership, group decision making, consensus, selforganization, speed-accuracy trade-off, goal orientation.

\section{Introduction}

Decision making in groups ranges from egalitarianism, where all individuals are involved, to leadership, where a few members (Strandburg-Peshkin et al. 2013) or even a single individual (Reebs 2000) can determine the group outcome. Although egalitarian (democratic, shared) group decision making should maximize utility for the group as

* Corresponding author; e-mail: c.c.ioannou@bristol.ac.uk.

Am. Nat. 2015. Vol. 186, pp. 284-293. (C) 2015 by The University of Chicago. 0003-0147/2015/18602-55866\$15.00. All rights reserved. DOI: $10.1086 / 681988$ a whole under a wide range of conditions (Conradt and Roper 2003), unequal (skewed) decisions are commonly observed (Krause et al. 2000; Van Vugt 2006; King 2010; Conradt 2012). Leadership can arise from individual differences in knowledge and experience (Reebs 2000; McComb et al. 2001; Couzin et al. 2005, 2011; Flack et al. 2012; Strandburg-Peshkin et al. 2013), energetic state (Rands et al. 2003; Fischhoff et al. 2007; Nakayama et al. 2012), and/or personality (e.g., boldness [Harcourt et al. 2009; Nakayama et al. 2012] and activity [Brown and Irving 2014]). Under some circumstances, processes of disproportionate influence can benefit all members of the group (McComb et al. 2001), and under others, leaders benefit relative to followers (Fischhoff et al. 2007). Leaders in many groups should be expected to maximize their individual gain without becoming spatially isolated from the group, which can expose them to an increased risk of predation (Ioannou et al. 2009, 2011a) and lose the informational benefits of group living (Berdahl et al. 2013; Miller et al. 2013). Due to the widespread nature of this form of leadership across taxa, the benefits of staying with the group often appear to outweigh the associated costs (Piyapong et al. 2007), such as longer travel times when leading compared to solitary movement (Franks and Richardson 2006).

The mechanisms underlying effective leadership have attracted a great deal of research in the social sciences (Van Vugt 2006; Haslam et al. 2010; King 2010). In animal groups, leadership is often studied in groups with dominance hierarchies, a stable composition, and/or relatively high relatedness between individuals, such as social insect colonies and primate societies (Seeley and Visscher 2004; Franks and Richardson 2006; King et al. 2008). However, leadership is also common in more loosely associated fission-fusion systems, where individuals have weaker persistent social ties and intergroup exchange commonly occurs (e.g., Reebs 2000; Huse et al. 2002; Lewis et al. 2011; 
Burns et al. 2012). Substantial differences in knowledge or experience are likely to exist in such groups, and as individuals experience fewer repeated interactions, they are likely to be less certain about the knowledge or behavioral traits of others (Biro et al. 2006). In the empirical studies with these conditions, research has instead focused on the mechanism(s) underlying information transfer, with the behavior of potential leaders either not measured and assumed to be constant (Pillot et al. 2011; Stienessen and Parrish 2013) or kept constant through the use of model conspecifics (Ward et al. 2008, although see Halloy et al. 2007 for an example where robot conspecifics can exert different degrees of influence depending on their behavioral parameters). Other studies, by contrast, have related variation in individuals' tendency to influence group decisions to behavioral or morphological traits (i.e., who tries to lead, rather than how; Reebs 2001; Harcourt et al. 2009; Schuett and Dall 2009; Freeman et al. 2011; Burns et al. 2012; Flack et al. 2012; Brown and Irving 2014).

In computational models of mobile self-organized animal groups, such as fish schools and bird flocks, some aspects of how organisms may balance goal directedness (e.g. a desire to move toward a known food source) with social cohesion have been explored. For example, in the framework of Couzin et al. (2005, 2011), Conradt et al. (2009), and Guttal and Couzin (2010), a simple, continuous weighting term, omega $(\omega)$, represents the strength to which each individual biases their motion toward a desired goal. If an individual's $\omega=0$, it does not express any goal directedness (this could correspond to individuals either lacking information regarding potential goals or being informed but completely discounting goal-directed behavior). If $\omega$ exceeds 0 , goal directedness begins to play a role in determining an individual's motion, with a value of 1 representing an equal balance between goal-directed and social tendencies. If $\omega$ increases beyond 1 , an individual's movement will be increasingly biased toward their desired direction of travel and, consequently, less influenced by social cues. Mathematical details of the equations of motion employed in these models and the implementation of $\omega$ can be found in the appendix, available online. Although increasing $\omega$ is predicted to exert greater influence on other group members (Couzin et al. 2005, 2011), it also increases the likelihood of isolation and thus of a corresponding failure to lead (Couzin et al. 2005; Conradt et al. 2009). Earlier empirical work has focused on groups with stable group membership, groups that have little opportunity to split, and/or only groups that remain cohesive (Partridge 1981; Seeley and Visscher 2004; Harcourt et al. 2009; Freeman et al. 2011), leaving these predictions largely untested.

To explore how differences in the behavior of trained individuals affect their ability to successfully lead others within mobile groups, we trained fish (golden shiners,
Notemigonus crysoleucas) to expect food in a dish in a corner of a large arena before testing them each singly with eight untrained fish. We trained fish to one of two identical dishes (in adjacent corners of a test tank), allowing us to assess the accuracy of fish in reaching the specific target to which they were trained. To remove potential confounds due to modification of behavior over repeated trials, each trained fish was tested only once with untrained fish. It is also unlikely that individuals were aware of the preferences of others in our experiments: trained fish were trained only with other trained fish until being tested, and untrained fish were unlikely to be aware of a knowledgeable leader. Trained and untrained fish were also obtained from separate stock tanks so that they were unfamiliar with one another. Information transfer in fish schools, such as those formed by golden shiners, is mediated through relative motion and positions of individuals, not through active signaling (Ioannou et al. 2011b; Katz et al. 2011; StrandburgPeshkin et al. 2013), meeting further assumptions of models of leadership in self-organized groups (Couzin et al. 2005, 2011). To support our experimental findings, we use a simple model to integrate experimental and theoretical insights and predictions.

\section{Methods}

Fish were sourced and kept under the same conditions as in Couzin et al. (2011). A total of 94 fish (mean standard body length $\pm \mathrm{SD}, 45 \pm 4.3 \mathrm{~mm}$ ) were each injected with an elastomer tag to differentiate trained from untrained fish. Five days later, they were moved to a $210 \times 120-\mathrm{cm}$ white acrylic glass test tank. The tank (fig. 1A) was split into sections using white plastic dividers: at one end, two $36 \times 35-\mathrm{cm}$ compartments held 47 fish each, between which was a start area $(35 \mathrm{~cm} \times 36.5 \mathrm{~cm})$. This led into a main area $(169 \mathrm{~cm} \times 120 \mathrm{~cm})$ via a remotely lifted door. In the main arena, a 54-mm petri dish surrounded by dark blue tape was positioned in each of the two corners opposite to the start area.

After habituating the fish to the main arena overnight and moving them to the holding compartments, the training program began. Tropical flake food was finely crushed and weighed before being suspended in water and pipetted into the petri dish that was assigned as the target for fish from that holding compartment (the other dish served as the target for fish from the other compartment). Nine or 10 fish were then haphazardly selected and netted to the start area. The door was raised after 5 min of habituation; once the fish had found and consumed all of the food reward, the trial was ended. To gradually train the fish, the groups received a single daily session on days 1-5 with $0.05 \mathrm{~g}$ of crushed food at the target. Then, from day 6 to day 12 , the amount of food per session was reduced to 

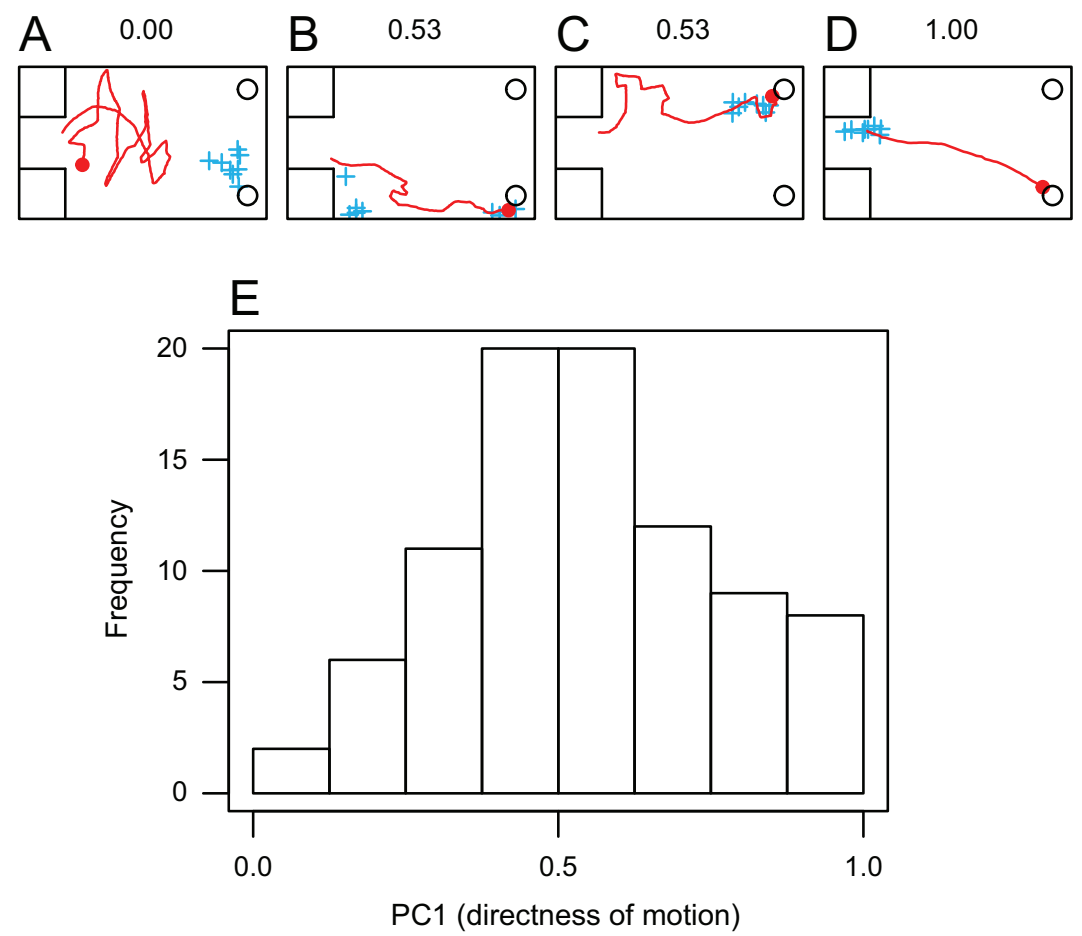

Figure 1: Variation in the directness of motion of trained fish when tested with untrained fish. $A-D$, Tank with examples of trained fish tracks (red lines) and positions of trained (red filled circles) and untrained (blue plus signs) fish at the end point of the trials. Also shown are the positions of the targets (black open circles) and the holding compartments in the other corners of the tank (for clarity, the raised door is not shown). $A-D$ correspond to the trials of trained fish with the lowest, two median, and greatest values for directness of motion, respectively, with the directness of motion value displayed above each panel. E, Overall distribution of the trained fish's directness of motion was approximately normally distributed. $\mathrm{PC} 1=$ first principal component.

$0.02 \mathrm{~g}$, and the procedure was repeated three times consecutively for each group. Once trials on that day were complete for a group, a transparent plastic barrier was used to partition the holding compartments to separate already trained fish from those yet to be trained. Training took place alternately between groups from the two compartments until all fish had participated, at which point the transparent barriers were removed. All fish were then fed crushed flake food $(\sim 0.65 \mathrm{~mL})$ and then fed again at the end of the day. If the procedure was started with fish from one compartment on one day, we started with fish from the other compartment on the following day to minimize any difference in training to the two different targets.

On the thirteenth day of training, testing with untrained fish began. A group of nine trained fish was given a single training trial (with $0.02 \mathrm{~g}$ of food) and returned to their partitioned holding compartment. A group of fish from the other compartment was then trained as on days 6-12. After these fish were returned to their holding compartment, eight uninformed (i.e., untrained) fish from one of three stock tanks were netted into the start area, along with a single fish from the first group trained that day (those tested only once). A minimum of $40 \mathrm{~min}$ had elapsed since the training of this first group. Similarly, the untrained fish had been fed at least $40 \mathrm{~min}$ before being moved to the experimental tank. The trial then proceeded using the same procedure as during training, although no food was present.

The main area was filmed during trials with a Canon XM2 camcorder at 25 frames per second, and a Panasonic HDC-HS300P camcorder filmed the start area and its immediate vicinity in the main area to allow the trained fish to be identified from the elastomer tag. Once either of the targets had been reached by a fish (i.e., when either a trained or untrained fish reached within two body lengths of a dish), the trial ended (Couzin et al. 2011). Deciding to end the trial at this point was due to the possibility (and likelihood, based on our observations) that the behavior of the fish upon reaching a dish gave feedback to the other fish as to the presence or absence of food. The trained fish was not reused, whereas the untrained fish were held separately until the end of testing that day, after which point they were returned to their source tank. This procedure was repeated for all the trained fish from that same subgroup of nine, using untrained fish from the same stock 
tank. After testing was complete, the remaining trained fish were trained as on days 6-12.

Over the following 9 days, this testing procedure was repeated until 45 fish from each compartment were tested. The three stock tanks ( 120 fish each) were used to supply untrained fish in a sequential order, so that an untrained fish could be used at most once every 3 days over a total testing period of 10 days. At this frequency of testing, and without food being present in these trials, it is highly unlikely that untrained fish learned to associate the dishes with food. All experiments were conducted in accordance with federal and state regulations and were approved by the Princeton University Institutional Animal Care and Use Committee.

The center-of-mass position of the trained fish was recorded manually (Rasband 2004) 25 times per second from leaving the start area. Of the 90 total trials with untrained fish, two were excluded from analysis: one where the video file was corrupted and the data were irretrievable and another where the trial was ended because an untrained fish reached a target before the trained fish left the start area, so tracking data could not be collected. The average speed, the coefficient of variation of the speed (a measure of acceleration and deceleration), and the path tortuosity (track length/distance between start and end points) were calculated for each trained fish's track. These three variables, in addition to the time taken for the trained fish to leave the start area, were included in a principal components analysis.

The first principal component (PC1) explained 54\% of the variance in trained fish behavior and had an eigenvalue of 1.47. This component correlated strongly with all variables (because absolute component loadings were greater than 0.42 in all cases; Tabachnick and Fidell 1996) except the time taken to leave the start area (component loading $=$ -0.21 ). The first principal component thus represents the directness of motion of a trained fish's path; in other words, straighter, faster, and less variable paths have larger values of PC1 (fig. $1 A-1 D$ ). This may correspond to the trained fish's degree of assertiveness and was normalized to range from 0 to 1 , with 0 being the least assertive trained fish and 1 being the most assertive trained fish. We also recorded the time taken for the first fish to leave the start area, which target was reached first, whether it was the trained fish that reached a target first, the center-of-mass position of each untrained fish at the end of the trial, and the total time taken (from the time the door was opened to a target being reached). In the first training session (i.e., before the trained fish were trained), the time taken for the first fish to leave the start area was recorded.

We used R 2.14.2 for all analyses (R Development Core Team 2011). For negative binomial and binomial generalized linear models (GLMs), the assumption of the disper- sion parameter being approximately equal to 1 was met or quasi distributions were used (see table A1 in the appendix). Polynomial and linear effects of PC1 on spacing between untrained fish were included after visually inspecting the trends. Statistics presented in the main text are with only PC1 as the explanatory variable, to reflect the fitted lines shown in the figures, whereas the effects of control variables as main effects (standard body length, target, number of days of training, and testing order in day) are presented in the appendix. Data for all presented analyses and plots are available from the Dryad Digital Repository: http://dx.doi.org/10.5061/dryad.qj07b (Ioannou et al. 2015).

To relate our experimental results to theory, we also conducted simulations of our experimental scenario using a generic and minimal spatial model for the study of collective dynamics in animal groups (adapted from Couzin et al. 2005, 2011). Both social interactions and goal-oriented behavior are included in this framework, with $\omega$ (a continuous term) representing the strength of goal-oriented behavior with respect to social influence (schooling tendency). Individuals with $\omega=0$ exhibit no directional preference and are thus equivalent to those who are untrained/ uninformed. As $\omega$ approaches 1, such individuals tend to equally balance social tendency with goal-directed behavior (in this case toward a target location), and if an individual's $\omega$ exceeds 1 , they are more strongly influenced by their preferred direction of travel (i.e., goal directedness) than by their social tendency. Consequently $\omega$ represents, for each individual, the balance between goal-oriented and social tendencies. To allow comparison with our experiment, we simulate nine individuals, of which eight have an $\omega$ of 0 (thus they exhibit no directional preference), and the potential leader has $\omega>0$, allowing us to explore the role of directional preference strength on leadership capability. A mathematical description and details of the model are provided in the appendix.

\section{Results \\ Effect of Training}

At the start of training, the first fish to leave the start area took between 29 and $445 \mathrm{~s}$ (mean $\pm \mathrm{SD}=210 \pm 136 \mathrm{~s}$; median $=175 \mathrm{~s} ; n=10$, because the 94 fish were trained in groups of nine or 10 fish). During testing with untrained fish, the trained fish appeared to initiate or contribute to the initiation of group movement in all trials included in the analysis; the trained individual was the first to leave the start area in 69 of the 88 trials, whereas, in the other 19 trials, the first fish left more quickly than the minimum time observed at the start of training.

Training reached a steady level by day 13 , as indicated by no significant effects of training duration while testing 
with untrained fish (table A1). Despite this, the paths of trained fish varied widely when tested with untrained fish. Some individuals showed no tendency toward the target to which they were trained (e.g., fig. $1 A$ ), whereas, at the other extreme, other individuals swam in a fast, straight trajectory to the target (e.g., fig. 1D). Overall, the directness of motion in trials with untrained fish was approximately normally distributed (fig. $1 E$ ), with most trained fish showing an intermediate directness of motion (e.g., fig. $1 B, 1 C)$.

\section{Accuracy: Reaching the Preferred Target}

We utilized the substantial variation shown by trained fish in their directness of motion to explore the features associated with effective leadership. As directness of motion increased, trained fish tended to be closer to their preferred target at the end of the trial (fig. 2). The likelihood (determined by likelihood ratio test [LRT]) that the preferred target was reached first by any fish increased with directness of motion of the trained fish $\left(\right.$ GLM: $\mathrm{LRT}_{1,86}=6.35$, $P=.012$ ). This was also the case when limiting the analy-

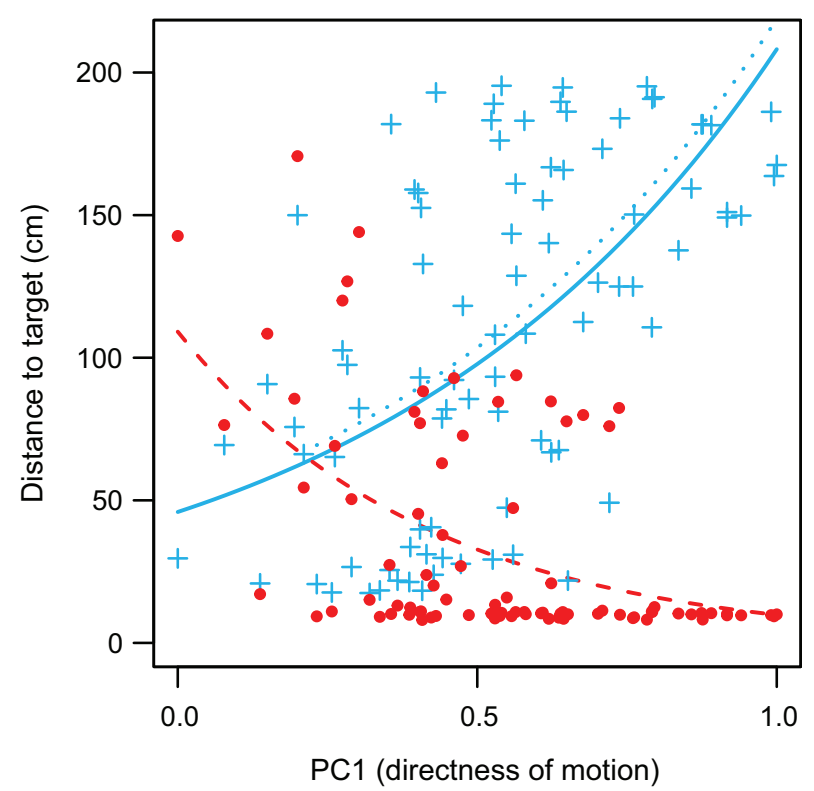

Figure 2: Proximity to the target for trained and untrained fish. As the trained fish's directness of motion increased, the trained fish got closer to the target at the end of the trial (red closed circles and dashed line: GLM, $\mathrm{LRT}_{1,86}=31.11, P=2.44 \times 10^{-8}$ ), whereas untrained fish were further away (mean of distances to the target for untrained fish: blue plus signs and solid line: $\mathrm{LRT}_{1,86}=24.74, P=$ $\left.6.56 \times 10^{-7}\right)$. Limiting this second analysis to only those trials where the preferred target was reached first by the trained fish yielded a very similar fit (blue dotted line, GLM: $\mathrm{LRT}_{1,48}=11.01, P=.00091$ ). $\mathrm{GLM}=$ generalized linear model; LRT = likelihood ratio test; PC1 = first principal component. sis to trials where the trained fish reached a target, either by reaching a target first or by being with the fish that reached the target first (see fig. 3 for definition; $n=81$; GLM: $\mathrm{LRT}_{1,79}=6.02, P=.014$ ). These results demonstrate that the directness of motion of the trained fish's path is a tendency toward the target that the fish was trained to (i.e., goal orientation), resulting in greater accuracy. Because faster and straighter paths are associated with reaching the preferred target, there exists a negative relationship between the total time taken (from the start of the trial to reaching a target) and the probability that the target reached was the one to which the trained fish was trained (for all trials, GLM: $\mathrm{LRT}_{1,86}=5.87, P=.015$; for only trials with trained fish reaching a target, GLM: $\left.\mathrm{LRT}_{1,79}=6.55, P=.011\right)$. In summary, there was a positive relationship between the speed and accuracy of the trained individual.

\section{Social Cohesion for Trained and Untrained Fish}

Although increasingly directed motion by trained fish was associated with speed and accuracy of reaching the target, it was also associated with failure to effectively lead untrained fish, resulting in greater nearest (GLM: LRT $_{1,86}=$ 17.81, $P=2.44 \times 10^{-5}$ ) and mean neighbor distances for the trained fish $\left(\right.$ GLM: $\left.\operatorname{LRT}_{1,86}=25.01, P=5.72 \times 10^{-7}\right)$. Correspondingly, untrained fish were farther from the target at the end of the trial when trained fish showed greater directness of motion (fig. 2). Even when the analyses were restricted to only those cases in which the trained fish reached the preferred target first (i.e., those individuals that showed a high tendency toward their target), these effects on potential followers remained (fig. $2 ; n=50$; for trained fish's nearest neighbor distance, GLM: $\mathrm{LRT}_{1,48}=7.43, P=$ .0064; for trained fish's mean distance to neighbors, GLM: $\left.\mathrm{LRT}_{1,48}=9.47, P=.0021\right)$. Trained fish thus appear to face a trade-off between the two components of effective leadership, as predicted from simulations (Couzin et al. 2005, 2011). Individuals showing more directed behavior reached their goal more effectively but potentially pay the cost of isolation by leaving followers behind (e.g., fig. 1D).

These results are consistent with a spring-like interaction between individuals (Katz et al. 2011) and support simulations showing that this interaction is breakable (Couzin et al. 2005). To investigate this further, instead of using the mean of the distances between the untrained and trained fish at the end of a trial, the distances between each untrained fish and the trained fish were examined and showed a bimodal distribution (fig. 3A). Untrained fish either stayed close to the trained individual or split from the potential leader, ending the trial far from the trained individual. Consistent with the nearest and mean distances between the untrained and trained fish, this outcome depended on the trained fish's directness of motion (fig. 3B). In 54 of the 

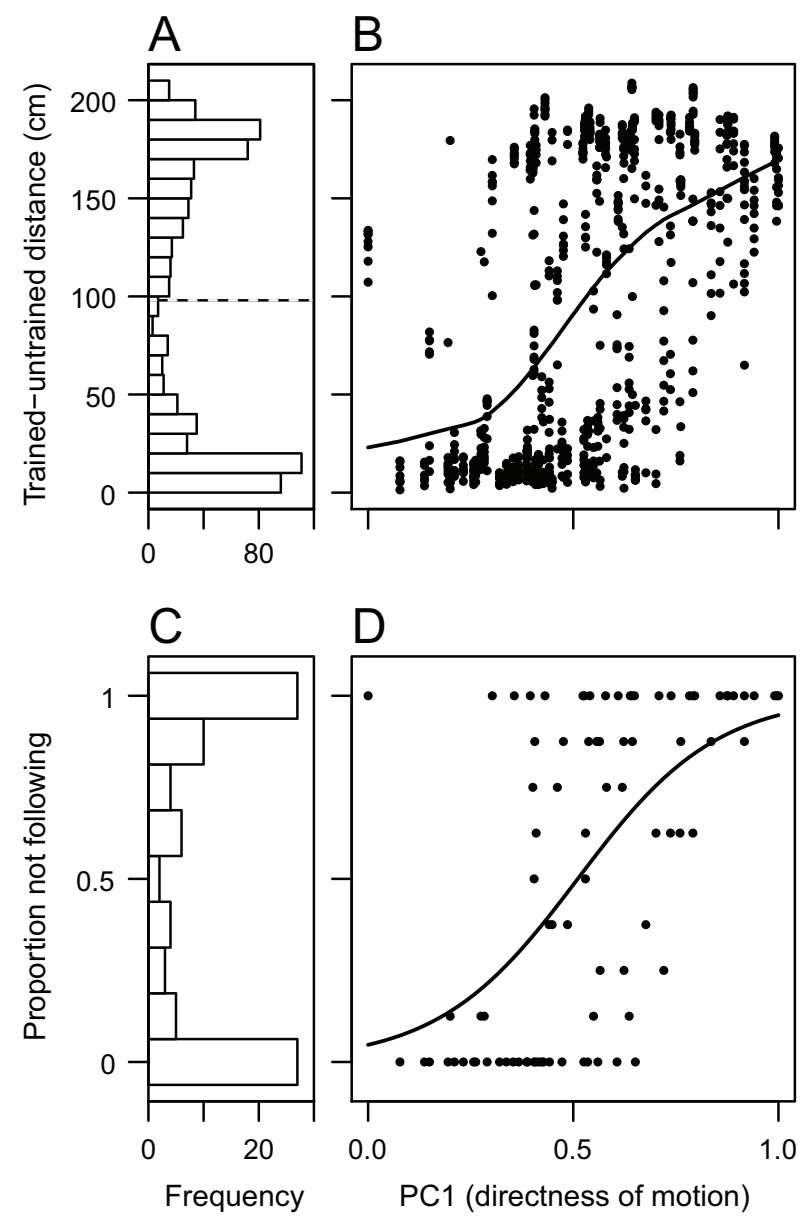

Figure 3: Distances between trained fish and untrained fish. A, Distribution of distances between individual untrained and trained fish, pooled over all trials, shows a clear bimodality. The dashed line indicates the mean of the distribution which is used to classify each untrained individual as either staying with or splitting from the trained individual to calculate the proportion of untrained fish in each trial that stayed with the trained fish $(C, D) . B$, It became more likely trained individuals would split away from the other fish as their directness of motion increased. The curve represents a locally weighted average using LOWESS ( $f$, the smoother span, is 0.625 ). $C$, Each untrained fish was defined as staying with or splitting from the trained fish depending on whether their distance to it was less or greater, respectively, than the mean in $A$. The proportion of untrained fish in each group staying with the trained individual shows a bimodal distribution as all eight untrained fish either stayed with or split from the trained individual. $D$, Corresponding to $B$, a greater proportion of the untrained fish split from the trained fish as the trained fish showed more directness of motion. PC1 $=$ first principal component.

88 trials, all eight untrained fish exhibited a strong tendency to effectively reach a consensus, either by all staying with the trained individual or by none of them doing so, resulting in a bimodal distribution overall (fig. 3C). The probability that the untrained fish split from the trained fish became greater as its directness of motion increased (fig. 3D; GLM: $\left.\mathrm{LRT}_{1,86}=34.99, P=6.55 \times 10^{-8}\right)$.

This property has not been investigated previously in simulation models. Although Couzin et al. (2005) predicted an association between increased goal-oriented behavior and group splitting, how the number of remaining followers related to the directness of motion of leaders was not reported. To determine whether our results are consistent with such models, we recreated our experimental scenario using the simulation framework described by Couzin et al. $(2005,2011)$, as described in the appendix. We found that, as in our experiments, increasing potential leaders' goal directedness $(\omega)$ resulted in them reaching the target to which they were trained more often (fig. A1A) and exhibiting more direct paths (fig. A1B) but that this came with the cost of failing to succeed in leadership. There exists a relatively abrupt transition between either all individuals or none of them tending to follow as leaders' $\omega$ increases (fig. A1C). As with our experimental findings, simulated potential leaders risked losing followers as their directness of motion increased (see appendix for details and model formulation).

The directness of motion of the trained fish was also found to be associated with cohesion between untrained fish. Unlike the distance between untrained and trained fish, however, untrained-untrained distances peaked at an intermediate level of the trained fish's directness of motion (fig. 4). This effect was statistically significant for mean and maximum neighbor distances (for mean, polynomial GLM: $\mathrm{LRT}_{2,85}=10.12, P=.0064$, and linear GLM: $\mathrm{LRT}_{1,86}=$ $0.84, P=.36$; for maximum, polynomial GLM: $\mathrm{LRT}_{2,85}=$ 9.28, $P=.0097$, and linear GLM: $\mathrm{LRT}_{1,86}=0.37, P=.54$ ) but not nearest neighbor distances (polynomial GLM: $\mathrm{LRT}_{2,85}=2.59, P=.27$, linear GLM: $\mathrm{LRT}_{1,86}=8.44 \times 10^{-5}$, $P=.99$ ). At intermediate levels of directness of motion, there was a trend for the maximum neighbor distance between untrained fish to be bimodal, with untrained fish splitting (high maximum distances) or not (low maximum distances) even at the same level of the trained fish's directness of motion (fig. $4 C$; as an example, see fig. $1 B, 1 C$, which shows the results of trials at the median level of directness of motion of the trained fish). This further supports the concept of a breakable social interaction (in this case, between untrained fish) being important in effective leadership.

\section{Effect of Trained Fish Behavior in Cohesive Groups}

In 27 of the 88 experimental trials, the untrained fish remained with the trained fish when a target was reached (i.e., the group, including trained fish, remained cohesive and did not split; fig. 3C). As in the analysis of all trials above, the target reached was more likely to be the trained 


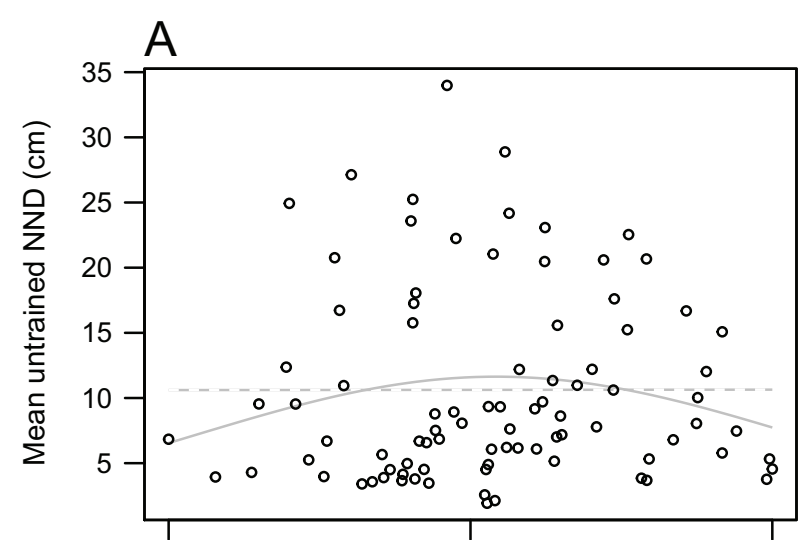

B
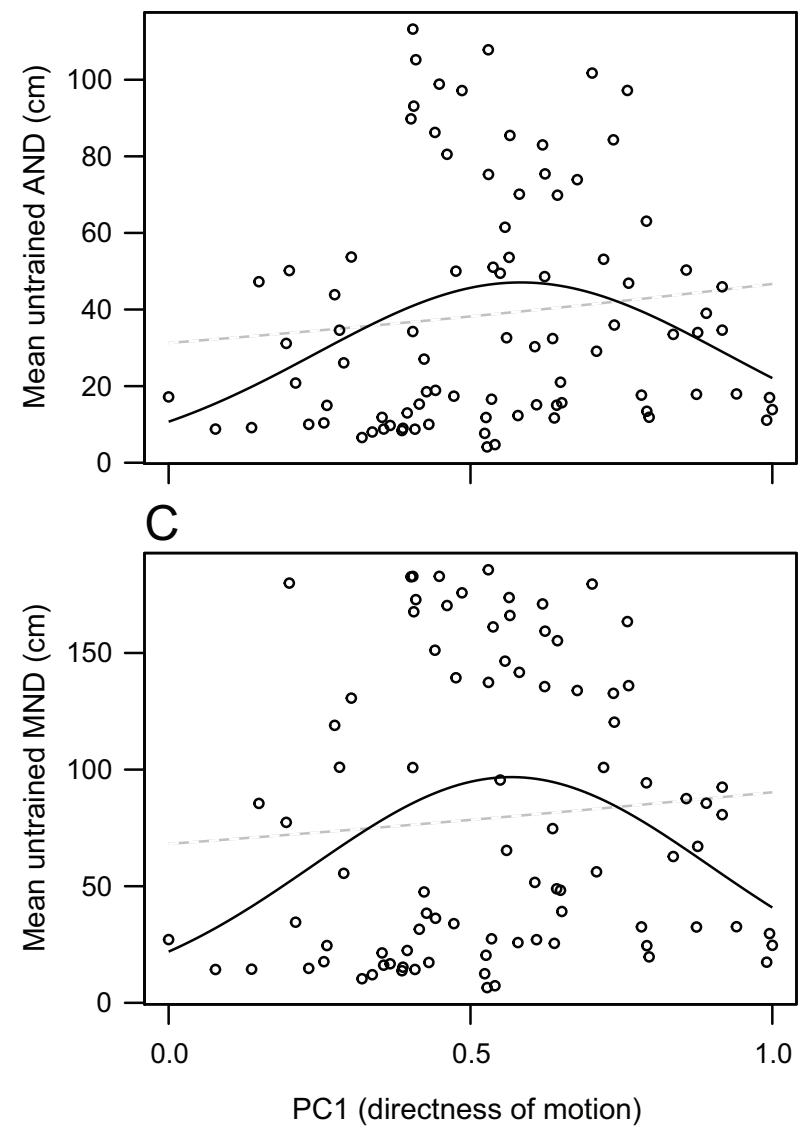

Figure 4: Distances between the untrained fish at the end of each trial as a function of the directness of motion of the trained fish. The nearest (NND; $A$ ), average (AND; $B$ ), and maximum (MND; $C$ ) neighbor distance is determined for each untrained fish, and the mean value is calculated across the eight untrained fish in the trial. Both linear (dashed lines) and polynomial (solid lines) fits to the data are shown, with significant trends at $P<.05$ in black and nonsignificant trends in gray. $\mathrm{PC} 1=$ first principal component. fish's preferred target when it showed more directed motion, although here, due to the reduced sample size, the effect is not significant (GLM: $\operatorname{LRT}_{1,25}=3.35, P=.067$ ). The strongly significant negative relationship between directness of motion and the total time taken for the trial remained significant (Spearman's rank correlation: $r_{\mathrm{s}}=$ $\left.-0.83, P=1.69 \times 10^{-6}\right)$, and the preferred target was more likely to be reached in faster trials (GLM: $\mathrm{LRT}_{1,25}=$ $4.64, P=.031)$. Thus, when untrained fish remain with the trained fish and the group is cohesive, the target is reached faster when the trained fish is more directed, and there remains a positive relationship between speed and accuracy.

\section{Discussion}

Leadership in both human and nonhuman animals is related to the ability to achieve a goal while maintaining coherence of group membership (Cartwright and Zander 1960; Seeley and Visscher 2004). In moving groups, this ability can be quantified as leadership accuracy, such as with respect to a preferred location, while ensuring that followers are not left behind (Couzin et al. 2005). Models have proved invaluable in revealing the general principles of collective behavior across seemingly disparate systems (Sumpter et al. 2012), but equally important is validating their core assumptions and predictions.

In our experiments, we found that, in accordance with theory, potential leaders (trained individuals) that exhibit highly directed motion toward the target do increase the accuracy and speed by which uninformed individuals are led to the correct target, but this behavior comes at the potential cost of completely failing to lead any individuals to that location. A similar effect has been observed in studies of social learning, where demonstrator individuals can be so highly trained that they separate from observers and fail to be followed (Swaney et al. 2001). Thus effective leadership in fish is consistent with theoretical predictions for self-organized groups, whereby potential leaders must be sufficiently assertive with respect to their preferred direction of travel but not so assertive that they risk isolation from the group.

Without testing each trained fish repeatedly, we could not assess whether the substantial variation in the trained fish's behavior was consistent between individuals over time, although a number of studies in a range of taxa demonstrate that this may be the case (e.g., Kurvers et al. 2009; Schuett and Dall 2009; Burns et al. 2012; Castanheira et al. 2013). The trade-off that informed individuals experience between reaching a target quickly and staying with a group will likely vary based on their willingness to risk isolation for increased rewards and is thus expected to vary with metabolic needs and perceived predation risk (Ioannou 
et al. 2008). Variation between individuals could be due to short-term plastic responses (e.g., responses to encountering a predator) or longer-term differences in perceived risk and food availability. Variation in these factors in untrained fish is also likely to be important, with more riskaverse or satiated individuals being harder to lead. In our experiment, individual differences that preexisted before training could also have become amplified if bolder fish took leader roles during training while other fish followed and became less well trained. This would be expected from previous work that shows that bolder individuals, with boldness being assessed before training, learn faster in terms of both speed and accuracy compared with shy individuals (Trompf and Brown 2014). We found that most trained fish showed motion consistent with an intermediate level of goal orientation when tested with untrained fish, rather than diverging into distinct assertive and nonassertive types, which suggests future modeling where the source and consequences of these distributions might be investigated (e.g., Guttal and Couzin 2010).

Individual variation is also expected to exist between the untrained fish with respect to their tendency to start exploring the arena (Ioannou et al. 2008; Castanheira et al. 2013), resulting in some fish having more influence on the school's movement than others. However, compared with the first training trials, where all fish were untrained, leaving the start area appeared to be influenced by the trained fish in all of the trials analyzed, which suggests that the tendencies of untrained fish were relatively weak compared with those observed in the trained fish. A recent study of information transfer, also involving schools of golden shiners, showed that untrained fish responded exclusively to social cues and not to the stimulus that trained fish were trained to respond to (Strandburg-Peshkin et al. 2013), and in other taxa, such as pigeons (Flack et al. 2012), training and experience can also dominate leadership beyond preexisting individual attributes. Thus, while untrained individuals could act as potential leaders, our results and those of Strandburg-Peshkin et al. (2013) suggest that this is unlikely.

A trade-off between speed and accuracy is frequently observed in the decisions made by isolated individuals and those made in groups (Lemasson et al. 2013; Trompf and Brown 2014). For many group-living animals, maintaining cohesion is an important dimension when decisions are made collectively (Sumpter and Pratt 2009; Miller et al. 2013). In many cases, such as decision making by social insects or primates, reaching consensus and hence maintaining group cohesion is the priority (Seeley and Visscher 2004), and the trade-off between decision speed and accuracy becomes "inevitable" (Sumpter and Pratt 2009). By contrast, the goal directedness of potential leaders, as found in our experiment, can instead result in a trade-off between staying with the group and making fast, accurate personal decisions. Recent experimental evidence shows that speed and accuracy can increase together during decision making by fish shoals, although this was associated with increased group size (Ward et al. 2011), rather than via a cost to a third variable (in this case, group cohesion).

In addition to the leader-follower relationship, we also identified a follower-follower interaction that was important in determining the outcome of attempted leadership. Social cohesion among untrained fish results in them typically forming a consensus as to whether to follow, with either all of them or none of them following the trained individual. Our simulations suggest that there need not exist an explicit mechanism for making this decision; such consensus behavior emerges spontaneously as a result of relatively local social interactions. Note that this process does not rely on the potential followers knowing that there is an informed fish present and their identity; as a potential leader's motion becomes increasingly goal directed, it is less social and more likely to split from others in the group. The higher social attraction of uninformed individuals maintains their cohesion, and hence generates the bimodal distribution of uninformed fish following or not following the informed fish. In our experiments, increased interindividual distances between untrained fish occurred only when the directness of motion of the trained fish was at an intermediate level, which suggests that the trained fish's influence was greatest at this point. These results support the existence of a synergy between leadership and selforganization (Couzin et al. 2005), with effective leadership associated with transmission of behavioral change across the group (social contagion of followership; StrandburgPeshkin et al. 2013). Bimodal responses driven by such forms of positive feedback have been shown repeatedly across taxa (e.g., Amé et al. 2006; Ward et al. 2008; Miller et al. 2013). In contrast to the overall group decision being led by a minority or collectively driven, these processes occur simultaneously (Couzin et al. 2005, 2011). Leaderfollower interactions will frequently be embedded in a larger network of follower-follower interactions, most obviously in groups with strong social ties (Jolles et al. 2013; Ward et al. 2013) but also in groups where individuals may not recognize one another.

As has been shown previously in humans (Haslam et al. 2010) and predicted in simulations (Couzin et al. 2005), we demonstrate empirically that effective leadership in animals depends on more than just a strong desire to achieve a goal. Any definition of successful leadership must include success in being followed (Krause et al. 2000; King 2010). Communication between leaders and followers relies on leaders balancing their desired tendency to reach a target with the risk of isolation from the rest of the group (Couzin et al. 2005), and we show that this does not necessarily rely 
on complex cognitive abilities, such as signaling and individual recognition. Although the mechanisms underlying information transfer within groups are clearly different between fish schools and human social organizations, and our method differs markedly from those used in the study of humans, the results presented here suggest that such a balance may be a fundamental property of leadership.

\section{Acknowledgments}

We thank S. Rands and the Couzin laboratory for helpful discussions and C. Brown, T. Day, S. O'Donnell, and an anonymous reviewer for comments on the manuscript. This work was funded by a Leverhulme Trust Early Career Fellowship (ECF-2011-628 to C.C.I.), a Natural Environment Research Council Independent Research Fellowship (NE/K009370/1 to C.C.I.), Office of Naval Research awards (N00014-09-1-1074 and N00014-14-1-0635 to I.D.C.), National Science Foundation awards (PHY-0848755, EAGER 1251585, and IOS 1355061 to I.D.C.), a Searle Scholar award (08-SPP-201 to I.D.C.), an Army Research Office grant (W911NG-11-1-0385 to I.D.C.), and a Human Frontier Science Program award (RGP0065/2012 to I.D.C.).

\section{Literature Cited}

Amé, J. M., J. Halloy, C. Rivault, C. Detrain, and J. L. Deneubourg. 2006. Collegial decision making based on social amplification leads to optimal group formation. Proceedings of the National Academy of Sciences of the USA 108:5835-5840.

Berdahl, A., C. J. Torney, C. C. Ioannou, J. J. Faria, and I. D. Couzin. 2013. Emergent sensing of complex environments by mobile animal groups. Science 339:574-576.

Biro, D., D. J. T. Sumpter, J. Meade, and T. Guilford. 2006. From compromise to leadership in pigeon homing. Current Biology 16: 2123-2128.

Brown, C., and E. Irving. 2014. Individual personality traits influence group exploration in a feral guppy population. Behavioral Ecology 25:95-101.

Burns, A. L. J., J. E. Herbert-Read, L. J. Morrell, and A. J. W. Ward. 2012. Consistency of leadership in shoals of mosquitofish (Gambusia holbrooki) in novel and in familiar environments. PLoS ONE 7:e36567.

Cartwright, D., and A. Zander. 1960. Group dynamics: research and theory. 2nd ed. Harper \& Row, New York.

Castanheira, M. F., M. Herrera, B. Costas, L. E. C. Conceição, and C. I. M. Martins. 2013. Can we predict personality in fish? searching for consistency over time and across contexts. PLoS ONE 8: e62037.

Conradt, L. 2012. Models in animal collective decision-making: information uncertainty and conflicting preferences. Interface Focus $2: 226-240$

Conradt, L., J. Krause, I. D. Couzin, and T. J. Roper. 2009. “Leading according to need" in self-organizing groups. American Naturalist 173:304-312.
Conradt, L., and T. J. Roper. 2003. Group decision-making in animals. Nature 421:155-158.

Couzin, I. D., C. C. Ioannou, G. Demirel, T. Gross, C. J. Torney, A. Hartnett, L. Conradt, et al. 2011. Uninformed individuals promote democratic consensus in animal groups. Science 334:15781580.

Couzin, I. D., J. Krause, N. R. Franks, and S. A. Levin. 2005. Effective leadership and decision-making in animal groups on the move. Nature 433:513-516.

Fischhoff, I. R., S. R. Sundaresan, J. Cordingley, H. M. Larkin, M.-J. J. Sellier, and D. I. Rubenstein. 2007. Social relationships and reproductive state influence leadership roles in movements of plains zebra, Equus burchellii. Animal Behaviour 73:825-831.

Flack, A., B. Pettit, R. Freeman, T. Guilford, and D. Biro. 2012. What are leaders made of? the role of individual experience in determining leader-follower relations in homing pigeons. Animal Behaviour 83:703-709.

Franks, N. R., and T. Richardson. 2006. Teaching in tandem-running ants. Nature 439:153.

Freeman, R., R. Mann, T. Guilford, and D. Biro. 2011. Group decisions and individual differences: route fidelity predicts flight leadership in homing pigeons (Columba livia). Biology Letters 7:63-66.

Guttal, V., and I. D. Couzin. 2010. Social interactions, information use, and the evolution of collective migration. Proceedings of the National Academy of Sciences of the USA 107:16172-16177.

Halloy, J., G. Sempo, G. Caprari, C. Rivault, M. Asadpour, F. Tache, I. Said, et al. 2007. Social integration of robots into groups of cockroaches to control self-organized choices. Science 318:1155-1158.

Harcourt, J. L., T. Z. Ang, G. Sweetman, R. A. Johnstone, and A. Manica. 2009. Social feedback and the emergence of leaders and followers. Current Biology 19:248-252.

Haslam, S. A., S. D. Reicher, and M. J. Platow. 2010. The new psychology of leadership: identity, influence and power. Psychology, New York.

Huse, G., S. Railsback, and A. Ferno. 2002. Modelling changes in migration pattern of herring: collective behaviour and numerical domination. Journal of Fish Biology 60:571-582.

Ioannou, C. C., F. Bartumeus, J. Krause, and G. D. Ruxton. $2011 a$. Unified effects of aggregation reveal larger prey groups take longer to find. Proceedings of the Royal Society B: Biological Sciences 278:2985-2990.

Ioannou, C. C., I. D. Couzin, R. James, D. P. Croft, and J. Krause. 2011b. Social organisation and information transfer in schooling fish. Pages 217-239 in C. Brown, K. Laland, and J. Krause, eds. Fish cognition and behavior. 2nd ed. Wiley-Blackwell, New York. Ioannou, C. C., L. J. Morrell, G. D. Ruxton, and J. Krause. 2009. The effect of prey density on predators: conspicuousness and attack success are sensitive to spatial scale. American Naturalist 173:499-506.

Ioannou, C. C., M. Payne, and J. Krause. 2008. Ecological consequences of the bold-shy continuum: the effect of predator boldness on prey risk. Oecologia (Berlin) 157:177-182.

Ioannou, C. C., M. Singh, and I. D. Couzin. 2015. Data from: Potential leaders trade off goal-oriented and socially oriented behavior in mobile animal groups. American Naturalist, Dryad Digital Repository, http://dx.doi.org/10.5061/dryad.qj07b.

Jolles, J. W., A. J. King, A. Manica, and A. Thornton. 2013. Heterogeneous structure in mixed-species corvid flocks in flight. Animal Behaviour 85:743-750.

Katz, Y., K. Tunstrøm, C. C. Ioannou, H. Huepe, and I. Couzin. 2011. Inferring the structure and dynamics of interactions in 
schooling fish. Proceedings of the National Academy of Sciences of the USA 108:18720-18725.

King, A. J. 2010. Follow me! I'm a leader if you do; I'm a failed initiator if you don't? Behavioural Processes 84:671-674.

King, A. J., C. Douglas, E. Huchard, N. J. B. Isaac, and G. Cowlishaw. 2008. Dominance and affiliation mediate despotism in a social primate. Current Biology 18:1833-1838.

Krause, J., D. Hoare, S. Krause, C. K. Hemelrijk, and D. I. Rubenstein. 2000. Leadership in fish shoals. Fish and Fisheries 1:82-89.

Kurvers, R. H. J. M., B. Eijkelenkamp, K. van Oers, B. van Lith, S. E. van Wieren, R. C. Ydenberg, and H. H. T. Prins. 2009. Personality differences explain leadership in barnacle geese. Animal Behaviour 78:447-453.

Lemasson, B. H., J. J. Anderson, and R. A. Goodwin. 2013. Motionguided attention promotes adaptive communications during social navigation. Proceedings of the Royal Society B: Biological Sciences 280:20122003.

Lewis, J. S., D. Wartzok, and M. R. Heithaus. 2011. Highly dynamic fission-fusion species can exhibit leadership when traveling. Behavioral Ecology and Sociobiology 65:1061-1069.

McComb, K., C. Moss, S. M. Durant, L. Baker, and S. Sayialel. 2001. Matriarchs as repositories of social knowledge in African elephants. Science 292:491-494.

Miller, N., S. Garnier, A. T. Hartnett, and I. D. Couzin. 2013. Both information and social cohesion determine collective decisions in animal groups. Proceedings of the National Academy of Sciences of the USA 110:5263-5268.

Nakayama, S., R. A. Johnstone, and A. Manica. 2012. Temperament and hunger interact to determine the emergence of leaders in pairs of foraging fish. PLoS ONE 7:e43747.

Partridge, B. L. 1981. Internal dynamics and the interrelations of fish in schools. Journal of Comparative Physiology A: Neuroethology, Sensory, Neural, and Behavioral Physiology 144:313-325.

Pillot, M. H., J. Gautrais, P. Arrufat, I. D. Couzin, R. Bon, and J. L. Deneubourg. 2011. Scalable rules for coherent group motion in a gregarious vertebrate. PLoS ONE 6:e14487.

Piyapong, C., L. J. Morrell, D. P. Croft, J. R. G. Dyer, C. C. Ioannou, and J. Krause. 2007. A cost of leadership in human groups. Ethology 113:821-824.

R Development Core Team. 2011. R: a language and environment for statistical computing. R Foundation for Statistical Computing, Vienna.

Rands, S. A., G. Cowlishaw, R. A. Pettifor, J. M. Rowcliffe, and R. A. Johnstone. 2003. Spontaneous emergence of leaders and followers in foraging pairs. Nature 423:432-434.

Rasband, W. S. 2004. Image J 1.34s. National Institutes of Health, Bethesda, MD.
Reebs, S. G. 2000. Can a minority of informed leaders determine the foraging movements of a fish shoal? Animal Behaviour 59:403409.

. 2001. Influence of body size on leadership in shoals of golden shiners, Notemigonus crysoleucas. Behaviour 138:797-809.

Schuett, W., and S. R. X. Dall. 2009. Sex differences, social context and personality in zebra finches, Taeniopygia guttata. Animal Behaviour 77:1041-1050.

Seeley, T. D., and P. K. Visscher. 2004. Group decision making in nest-site selection by honey bees. Apidologie 35:101-116.

Stienessen, S. C., and J. K. Parrish. 2013. The effect of disparate information on individual fish movements and emergent group behavior. Behavioral Ecology 24:1150-1160.

Strandburg-Peshkin, A., C. R. Twomey, N. W. F. Bode, A. B. Kao, Y. Katz, C. C. Ioannou, S. B. Rosenthal, et al. 2013. Visual sensory networks and effective information transfer in animal groups. Current Biology 23:R709-R711.

Sumpter, D. J. T., R. P. Mann, and A. Perna. 2012. The modelling cycle for collective animal behaviour. Interface Focus 2:764-773.

Sumpter, D. J. T., and S. C. Pratt. 2009. Quorum responses and consensus decision making. Philosophical Transactions of the Royal Society B: Biological Sciences 364:743-753.

Swaney, W., J. Kendal, H. Capon, C. Brown, and K. N. Laland. 2001. Familiarity facilitates social learning of foraging behaviour in the guppy. Animal Behaviour 62:591-598.

Tabachnick, B. G., and L. S. Fidell. 1996. Using multivariate statistics. Harper Collins, New York.

Trompf, L., and C. Brown. 2014. Personality affects learning and trade-offs between private and social information in guppies, Poecilia reticulata. Animal Behaviour 88:99-106.

Van Vugt, M. 2006. Evolutionary origins of leadership and followership. Personality and Social Psychology Review 10:354-371.

Ward, A. J. W., J. E. Herbert-Read, L. A. Jordan, R. James, J. Krause, Q. Ma, D. I. Rubenstein, et al. 2013. Initiators, leaders, and recruitment mechanisms in the collective movements of damselfish. American Naturalist 181:748-760.

Ward, A. J. W., J. E. Herbert-Read, D. J. T. Sumpter, and J. Krause. 2011. Fast and accurate decisions through collective vigilance in fish shoals. Proceedings of the National Academy of Sciences of the USA 108:2312-2315.

Ward, A. J. W., D. J. T. Sumpter, I. D. Couzin, P. J. B. Hart, and J. Krause. 2008. Quorum decision-making facilitates information transfer in fish shoals. Proceedings of the National Academy of Sciences of the USA 105:6948-6953.

Associate Editor: Sean O'Donnell Editor: Troy Day 